\title{
Multiparametrische MR-Bildgebung beim Prostatakarzinom
}

Heinz-Peter Schlemmer

\section{Über die multiparametrische Prostata-MRT (mpMRT) ist viel geschrieben worden. Richtig durchgeführt, kann sie die Diagnostik verbessern, erlaubt eine zuverläs- sigere Prognoseabschätzung und hilft dabei, die individuell optimale Therapie festzulegen. Ziel (und Herausforderung) dieser kurzen Übersicht ist es, Radiolo- gen das nötigste methodische Handwerkszeug und Hintergrundwissen für die tägliche Routine an die Hand zu geben.}

\section{Einleitung}

Das Prostatakarzinom ist eine große medizinische und sozioökonomische Herausforderung [1]. Hohe Inzidenz, große Variabilität der Tumoraggressivität, unpräzise Diagnostik und unzureichende Individualisierung der Therapie mit Überbehandlung wenig aggressiver Karzinome kennzeichnen die derzeitige Situation [25]. Dabei ist das Prostatakarzinom in den Industrienationen die häufigste Krebserkrankung des Mannes. In Deutschland rechnet man derzeit pro Jahr mit etwa 70000 Neuerkrankungen und 12000 Todesfällen (Platz 3 der Krebsletalität).

Größter Risikofaktor ist das Alter mit stark ansteigender Erkrankungshäufigkeit ab dem 55. Lebensjahr und Maximum etwa im 70. Lebensjahr. Epidemiologische Untersuchungen weisen zudem auf eine genetische Komponente hin, da familiäre Häufungen sowie starke geografische und ethnische Unterschiede beobachtet werden $[6,7]$.

Die konventionelle urologische Diagnostik zur Früherkennung besteht aus digitaler rektaler Untersuchung (DRU), PSA-Serumtest und - abhängig hiervon - systematischer Prostatabiopsie (üblicherweise transrektale 12-fach-TRUS-Biopsie). Problematisch ist, dass erhöhte PSA-Serumwerte auch Folge der viel häufigeren benignen Prostatahyperplasie (BPH) und Prostatitis sind [8]. Der Schwellenwert für eine normale PSA-Serumkonzentration liegt - je nach Alter - zwischen etwa $2,5 \mathrm{ng} / \mathrm{ml}$ und $4 \mathrm{ng} / \mathrm{ml}$. Diagnostisch und prognostisch relevant sind weitere PSA-Parameter wie die PSA-Dichte (relativ zum Prostatavolumen), das freie PSA und die sog. PSA-Dynamik, d.h. der Zeitverlauf der Serumkonzentration. Die PSA-Parameter werden von den Urologen in Abhängigkeit von u. a. Alter, Prostatagröße und evtl. vorhandenen benignen Prostataerkrankungen differenziert interpretiert.

\begin{abstract}
FALLBEISPIEL
Erhöhter PSA-Wert

Eine Vorsorgeuntersuchung bei einem 56 Jahre alten Mann ergab beim Hausarzt einen PSA-Wert von $6,4 \mathrm{ng} / \mathrm{ml}$. Die digitale rektale Untersuchung war unauffällig. Der Patient gab an, keine urologischen Beschwerden zu haben, eine Diagnostik der Prostata, insbesondere eine Prostatabiopsie, hatte bis zu diesem Zeitpunkt nicht stattgefunden. Der Hausarzt überwies den Patienten zur Abklärung.

Es wurde eine multiparametrische Prostata-MRT durchgeführt, um karzinomsuspekte Herdbefunde nachzuweisen oder auszuschließen, und in Abhängigkeit vom Ergebnis ggf. eine Biopsie zu planen.
\end{abstract}

Mit einer korrekt durchgeführten mpMRT der Prostata ist es nachweislich möglich, die Diagnostik und das lokale Staging zu verbessern, die Prognoseeinschätzung auf eine zuverlässigere Grundlage zu stellen und die Therapie zu individualisieren. In den letzten Jahren hat insbesondere die Einführung der mpMRT-gezielten Biopsie die primäre Diagnostik verbessert.

\section{Merke}

Mit modernen MR-Tomografen ist die mpMRT in der Praxis möglich, ohne dass dafür spezielles Equipment oder besondere Bildnachverarbeitungsverfahren nötig wären. 


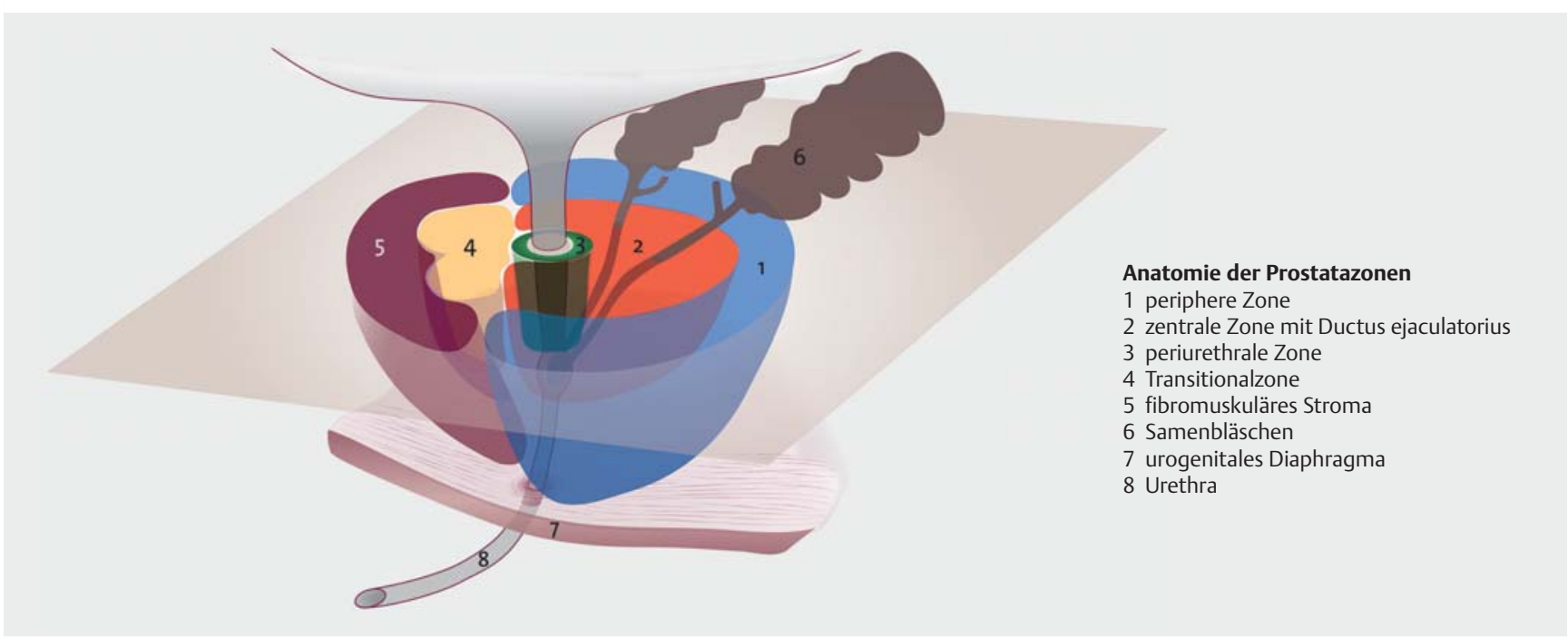

- Abb. 1 Skizze des zonalen Aufbaus der Prostata. In etwa der Mitte der intraprostatischen Urethra liegt der Colliculus seminalis. In diesen münden von dorsal her die beiden Ductus ejaculatorii ein. Ein Ductus ejaculatorius ist die Zusammenführung des distalen Samenleiters (Ampulla ductus deferentis) und des Ausführungsgangs der Samenblasen (Ductus excretorius). Die beiden Ductus ejaculatorii sind von der zentralen Zone (2) umgeben. Ventral hiervon liegt die paarig angelegte Transitionalzone (4). Die schmale periurethrale Zone (3) umgibt die intraprostatische Harnröhre zwischen dem Blasenhals und dem Colliculus. Die peripherere Zone (1) befindet sich vorwiegend dorsal/lateral und nimmt an Volumen zum Apex hin zu. Die Zone fibromuskulären Stromas (anteriores fibromuskuläres Stroma, 5) befindet sich ventral/basal. Von den Drüsenanteilen der Prostata enthält die periphere Zone ca. 75 \%, die zentrale Zone 20\% und die Transitionalzone $5 \%$.

Für die bestmögliche Patientenversorgung müssen aber folgende Voraussetzungen erfüllt sein:

- hohe Qualität und Standardisierung der Untersuchung

- ausreichend hohe Expertise bei der Bildbeurteilung

- standardisierte Befunddokumentation und Befundübermittlung nach PI-RADS

- intensive interdisziplinäre Zusammenarbeit von Urologen und Radiologen, um die Qualität fortlaufend zu kontrollieren und nachhaltig zu verbessern

\section{Anatomie der Prostata}

Die exakte Kenntnis der Anatomie der Prostata mit dem nach McNeal benannten zonalen Aufbau ist von grundlegender Bedeutung für die korrekte Bildinterpretation der mpMRT $[9,10]$. Die Prostata liegt zwischen Harnblase und Peniswurzel und hat die Form einer umgekehrten Pyramide mit Basis kranial am Harnblasenboden und Spitze (Apex) am Beckenboden ( $\triangleright$ Abb. 1). Bis etwa zum 45. Lebensjahr ist sie in der Transversalebene ca. $4 \mathrm{~cm}$ (rechts-links) $\times 3 \mathrm{~cm}$ (anterior-posterior) und in kraniokaudaler Richtung $3 \mathrm{~cm}$ groß. Die Drüse umschließt den sog. intraprostatischen Anteil der Harnröhre (Pars intraprostatica), in den die Ausführungsgänge von etwa 30 Prostatadrüsen einmünden. In etwa der Mitte der intraprostatischen Urethra liegt der Colliculus seminalis. In diesen münden von dorsal her die beiden Ductus ejaculatorii ein, die jeweils die Ampulla ductus deferentis, d. h. den distalen Samenleiter, mit dem Ductus excretorius der Samenblasen verbinden.
Die beiden Ductus durchziehen die Prostata von der Basis her und sind im Verlauf von der zentralen Zone (CZ) umgeben. Ventral der CZ liegt die paarig angelegte Transitionalzone (TZ). Die TZ ist der Entstehungsort der benignen Prostatahyperplasie (BPH), die im Alter zu der bekannten, teils erheblichen knotigen Vergrößerung der Drüse führt. Die intraprostatische Harnröhre selbst ist zwischen dem Blasenhals und dem Colliculus von einer schmalen, bildmorphologisch jedoch kaum abgrenzbaren Mantelzone, der sog. periurethralen Zone umgeben. Periurethrale, transitionale und zentrale Zone werden manchmal als „zentrale Drüse“ zusammenfasst, da die einzelnen Zonen bildmorphologisch schwer zu trennen sind. Dieser Begriff sollte aber vermieden werden, weil der größte Anteil dieses vergrößerten Drüsenabschnitts aus der vergrößerten TZ besteht und die (nicht vergrößerte) CZ nach jeweils lateral verdrängt ist. Die peripherere Zone (PZ) befindet sich vorwiegend dorsal sowie lateral und nimmt zum Apex hin an Volumen zu, wo sie die Urethra zirkulär umfasst.

\section{Merke}

Von den Drüsenanteilen der Prostata enthält die PZ ca. $75 \%$, die CZ $20 \%$ und die TZ $5 \%$.

Die Zone fibromuskulären Stromas (anteriores fibromuskuläres Stroma, AFS) befindet sich vorwiegend basal, ventral und median symmetrisch. 


\section{Multiparametrische MRT}

Die mpMRT der Prostata hat in erster Linie die Aufgabe, karzinomsuspekte Herdbefunde nachzuweisen, zu lokalisieren und zu dokumentieren. Im Fall eines bereits bewiesenen Karzinoms setzt man die mpMRT für das Tumorstaging ein. Der Befund liefert dem Urologen und seinem Patienten eine wichtige Entscheidungsgrundlage für das weitere diagnostische und therapeutische Vorgehen (z. B. bildgebungsgestützte Biopsie, Therapieplanung, Active Surveillance). Für eine Routineanwendung der mpMRT sind allerdings objektive Standards erforderlich, mit denen man die Untersuchung durchführt und die Befunde interpretiert, dokumentiert und kommuniziert. Ein besonderes Augenmerk sollte auch der fehlerfreien Schnittstelle zum überweisenden Urologen geschenkt werden.

\section{Klinische Informationen}

Für die Bildbeurteilung sollten folgende Informationen vorliegen:

- aktueller PSA-Serumwert und, falls vorhanden, dessen zeitlicher Verlauf (PSA-Dynamik)

- weitere Prostataerkrankungen (z.B. BPH, akute/ chronische Prostatitis) und deren Vorbehandlungen (z. B. transurethrale Prostataresektion, Antibiotikabehandlung)

- Familienanamnese (an Prostatakarzinom erkrankte Familienmitglieder)

- vorangehende Prostatabiopsien (Anzahl, Zeitpunkt) sowie deren pathologisches Ergebnis (Anzahl und Lokalisation der entnommenen Stanzzylinder, jeweils ggf. mit Gleason-Score und prozentualem Tumoranteil im Gewebezylindern)

\section{Vorbereitung}

Bei einer vorherigen Prostatabiopsie wird bis zur mpMRT ein Intervall von mindestens 6 Wochen empfohlen, um den Nachweis eines Tumors nicht durch postbioptische Einblutungen und/oder Entzündungen zu verhindern. Die Länge dieses Zeitintervalls ist aber umstritten, denn sollte sich in einem eingebluteten (d.h. dementsprechend auch biopsierten) Areal auch kein Herdbefund auf DWI- und DCE-Aufnahmen darstellen, so ist die Wahrscheinlichkeit, dass in diesem Areal ein klinisch signifikantes Karzinom vorliegt, sehr gering.

Die Empfehlung, Ejakulationen für mindestens 3 Tage vor der Untersuchung zu unterlassen, hat sich als diagnostisch nicht relevant herausgestellt.
Vor der Untersuchung sollte der Patient Harnblase und Rektum entleeren. Antiperistaltika (Buscopan, Glukagon) braucht man nicht regelhaft zu geben, sondern kann sie auf Einzelfälle mit ausgeprägter Darmperistaltik beschränken. Vorteilhaft ist dabei, dass mögliche Nebenwirkungen vermieden und Kosten reduziert werden.

\section{PRAXISTIPP}

\section{Vorbereitung des Patienten}

- mindestens 6 Wochen Abstand zur letzten Prostatabiopsie (wenn möglich)

- Harnblase und Rektum entleeren lassen

- Antiperistaltika nur bei Patienten mit ausgeprägter Darmperistaltik

\section{Feldstärke}

Das multiparametrische Untersuchungsprotokoll sollte am jeweiligen MR-Tomografen speziell optimiert werden. Das gilt insbesondere für die diffusionsgewichtete MRT, die mit hohen b-Werten den wesentlichen Kontrast für den Nachweis des Prostatakarzinoms in der PZ liefert. Aufgrund des besseren Signal-zu-RauschVerhältnisses (SNR) ist generell die Untersuchung bei 3,0 Tesla gegenüber der bei 1,5 Tesla zu bevorzugen. Untersucht wird i.d.R. mit der Phased-Array-Oberflächenspule. Ein ausreichend hohes SNR kann damit auch an modernen 1,5-Tesla-MR-Tomografen mit optimierten Sequenzprotokollen erzielt werden. Die Untersuchung bei 1,5 Tesla kann sogar von Vorteil sein, um suszeptibilitätsbedingte Artefakte durch Metallimplantate zu vermeiden. Von Relevanz ist dies insbesondere für die Qualität der DWI, die mit suszeptibilitätsempfindlichen EPI-Sequenzen aufgenommen wird. Auch sind manche Implantate grundsätzlich nur für Untersuchungen bis 1,5 Tesla zugelassen. Die Verwendung einer Endorektalspule ist nur noch bei älteren 1,5-TeslaTomografen mit unzureichendem SNR erforderlich. An Bedeutung verloren hat sie auch deshalb, weil die signalempfindliche MR-spektroskopische Bildgebung nur noch optionaler Bestandteil der mpMRT ist. Die Endorektalspule kann sogar für Artefakte verantwortlich sein, die durch die Kompression der Prostata, den Intensitätssprung an der Grenzfläche zur Prostata und durch Suszeptibilitätsartefakte infolge der Luftfüllung der Spule hervorgerufen werden. 
- Tab. 1 Untersuchungsprotokoll der multiparametrischen MRT der Prostata mit Kombination der 3 Sequenzen T2w, DWI und DCE. Der klinische Stellenwert der DCE wird derzeit noch kontrovers diskutiert. Die Schichtausrichtung erfolgt axial senkrecht zur Grenzlinie Prostata - Rektum, und in senkrechtem Winkel hierzu sagittal und koronar.

\begin{tabular}{|c|c|c|c|}
\hline MR-Sequenz & Korrelat & Parameter & Karzinomsuspekte Befunde=fokale Läsionen \\
\hline $\mathrm{T} 2 \mathrm{w}$ & Morphologie & $\begin{array}{l}\text { - } \text { axial, koronar, sagittal } \\
\text { - } 2 \text { D FSE/TSE } \\
\text { - } \text { TR/TE } 4000-8000 / 100-120 \\
\text { - SD } 3 \mathrm{~mm}, \varnothing \mathrm{Gap} \\
\text { - } \text { VS } \leq 0,7 \times 0,7 \mathrm{~mm}^{2}\end{array}$ & hypointenses Signal \\
\hline DWI & Zelldichte & $\begin{array}{l}\text { - } \text { axial } \\
\text { - SD } 3-4 \mathrm{~mm}, \varnothing \text { Gap } \\
\text { - b-Werte } \\
\text { - Standard }=50-100+1000 \mathrm{~s} / \mathrm{mm}^{2 *} \\
\text { - optional hohe b-Werte }=1400-2000 \mathrm{~s} / \mathrm{mm}^{2}\end{array}$ & $\begin{array}{l}\text { hyperintenses Signal bei hohen b-Werten und } \\
\text { niedriges Signal auf ADC-Karte }\end{array}$ \\
\hline DCE & Durchblutung & $\begin{array}{l}\text { - axial } \\
\text { - SD } 3 \mathrm{~mm}, \varnothing \text { Gap } \\
\text { - VS } \leq 0,2 \times 0,2 \mathrm{~mm}^{2} \\
\text { - Gd-haltiges Kontrastmittel: } \\
\text { - Standarddosierung } \\
\text { - Injektionsrate } 2-3 \mathrm{cc} / \mathrm{s} \\
\text { - Zeitauflösung } \leq \mathrm{ca} .10 \mathrm{~s} \\
\text { - Aufnahmezeit } \geq 2 \mathrm{~min}\end{array}$ & $\begin{array}{l}\text { früharterielles und starkes Kontrastmittelenhance- } \\
\text { ment }\end{array}$ \\
\hline $\begin{array}{l}\text { T1w und/oder } \\
\text { T2w, DWI }\end{array}$ & Morphologie & $\begin{array}{l}\text { " axial } \\
\text { - untere LWS-Beckenboden } \\
\text { " SD } 3-4 \mathrm{~mm} \\
\text { " großes FOV }\end{array}$ & $\begin{array}{l}\text { suspekte Morphologie/Signalintensität von ins- } \\
\text { besondere Lymphknoten, Knochenmark; Einblutung } \\
\text { in der Prostata }\end{array}$ \\
\hline
\end{tabular}

\section{Untersuchungsprotokoll und MR-Kontraste}

Die mpMRT setzt sich aus 3 wesentlichen Sequenzen bzw. Kontrastparametern zusammen:

- T2w MRT in axialer, koronarer und sagittaler Schnittführung zur Beurteilung der Morphologie

- DWI= diffusionsgewichtete MRT in axialer Schnittführung zur Beurteilung der Zelldichte

- $\mathrm{DCE}=$ kontrastmittelangehobene, dynamische MRT in axialer Schnittführung zur Beurteilung der Durchblutung (Neovaskularisation)

Das FOV muss jeweils klein und auf die Prostata und Samenblasen fokussiert sein. Die axiale Schichtführung sollte bei allen Sequenzen hinsichtlich Angulierung (senkrecht zur Grenzfläche Prostata-Rektum), Schichtposition und Schichtdicke übereinstimmen. Empfohlene Sequenzparameter sind in $>$ Tab. 1 aufgelistet. Die Arbeitsgemeinschaft Uroradiologie der Deutschen Röntgengesellschaft hat kürzlich eine aktuelle Empfehlung zur Prostata-MRT publiziert [11].

\section{T2w MRT}

Die normale periphere Zone stellt sich mit hohem T2Signal dar ( Abb. 2). Eine Signalabsenkung ist unspezifisch und wird sowohl beim Prostatakarzinom als auch bei verschiedenen benignen Erkrankungen (z. B. Prostatitis, stromale BHP, Atrophie, Fibrose) oder bei antihormoneller Behandlung gefunden. Dies schränkt den
Nachweis eines Prostatakarzinoms alleine auf Grundlage des T2-Signals erheblich ein. Die T2w Aufnahme ist jedoch die wichtigste Sequenz für das lokale Staging, denn im Normalzustand ist das T2-Signal sowohl des umgebenden periprostatischen Weichteilgewebes als auch der Samenblasen auf den (nicht fettunterdrückten) T2w Aufnahmen hoch. Eine Tumorausbreitung lässt sich daher an der charakteristischen, die Prostata überschreitenden Signalabsenkung erkennen.

\section{Diffusionsgewichtete MRT}

Die DWI liefert den wichtigsten Kontrast zum Nachweis des Prostatakarzinoms. Dabei ist die Wassermobilität im Fall eines Karzinoms sowohl im extrazellulären Raum (erhöhte Zelldichte) als auch im intrazellulären Raum (veränderte Matrix) vermindert. Ein ausreichendes SNR bei hohen b-Werten ist für die Qualität einer mpMRT wesentlich. Der ADC-Wert kann auf Grundlage der Signale bei niedrigem b-Wert $\left(b=50-100 \mathrm{~s} / \mathrm{mm}^{2}\right.$ zum Ausschluss von Perfusionseinflüssen) und einem hohen b-Wert $\left(\geq 1000 \mathrm{~s} / \mathrm{mm}^{2}\right)$ berechnet werden. Detaillierte Messmethoden mit Messung mehrerer b-Werte und differenzierte multiexponentielle Signalanalysen (IVIM und Kurtosis) sind Gegenstand der Wissenschaft und für die Routine nicht erforderlich. 


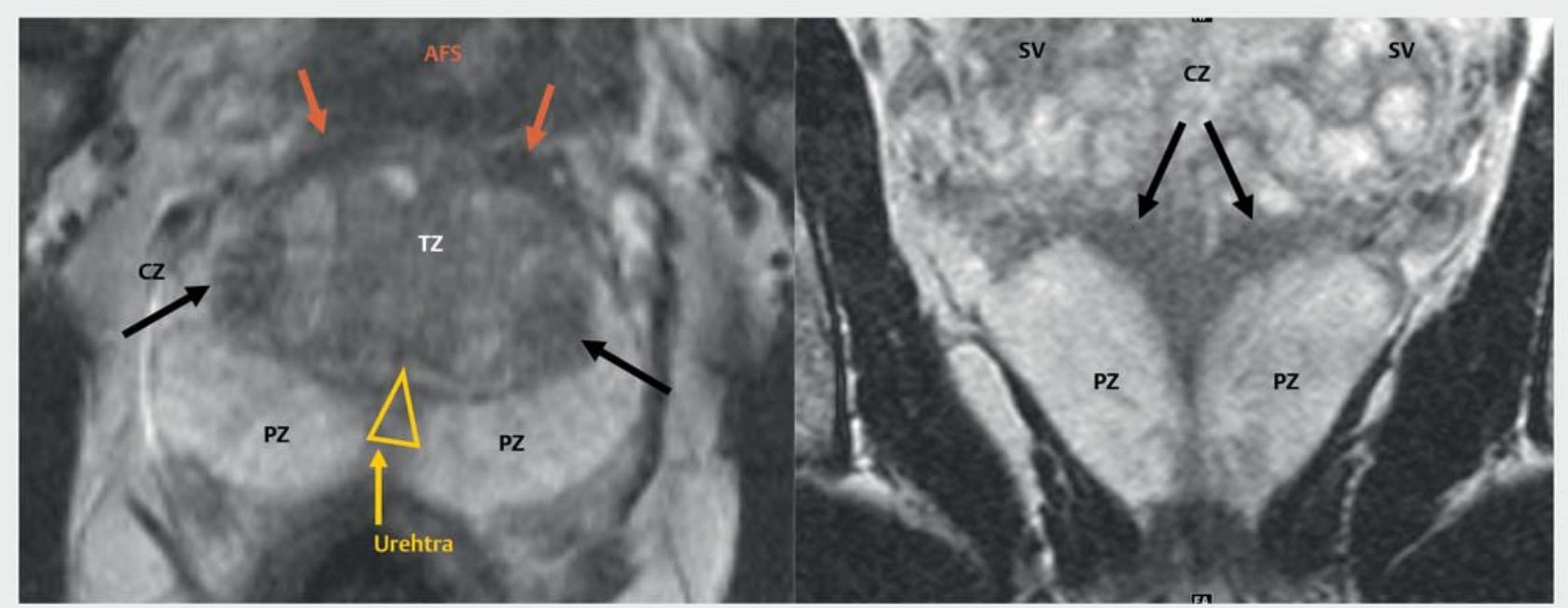

- Abb. 2 Häufiger Befund einer Prostata-MRT. Die periphere Zone (PZ) weist eine homogen hyperintense Signalgebung auf T2w Aufnahmen auf. Die Transitionalzone (TZ) ist durch eine BPH mit gemischter Signalintensität aufgrund unterschiedlicher glandulärer und stromaler Zusammensetzung vergrößert. Die TZ verdrängt dabei die zentrale Zone (CZ) nach lateral. Die Hypointensität (auf T2w Aufnahmen) der CZ sowie des anterioren fibromuskulären Stromas (AFS) darf nicht mit einem Prostatakarzinom verwechselt werden (typisches „Pitfall“). SV seminal vesicles.

Kontrastmittelangehobene, dynamische MRT

Ziel ist der Nachweis von Arealen mit starker früharterieller Kontrastmittelaufnahme („Wash-in“). Ein zusätzliches „Wash-out“ ist diagnostisch von untergeordneter Bedeutung. Die dynamische T1w-Sequenz und die Kontrastmittelgabe sollten gleichzeitig beginnen, wodurch native Aufnahmen automatisch zur Verfügung stehen. Die computerunterstützte Signalanalyse unter Verwendung pharmakokinetischer Modelle (z. B. Tofts, Brix) kann hilfreich sein, um Areale mit auffälligem Wash-in und Wash-out mittels farbcodierter Parameterkarten (semi)quantitativ zu objektivieren und zu visualisieren. Die Datennachverarbeitung ist aber mathematisch komplex (arterielle Inputfunktion, Kompartimente und Kontrastmittelaustauschraten) und hat sich für die Routineanwendung nicht als zwingend notwendig herausgestellt.

Eine früharterielle Kontrastierung gegenüber normalem Drüsengewebe der PZ ist charakteristisch für Prostatakarzinome (Neovaskularisation) - aber nicht spezifisch. Zwar geht die Prostatitis in der PZ häufig mit einer langsameren und flächigen Kontrastmittelaufnahme einher, aber stromale BPH-Knoten weisen in der TZ ebenso eine umschriebene früharterielle Mehrdurchblutung auf. Umgekehrt zeigen niedriggradige Karzinome ohne besondere Neovaskularisation keine typische früharterielle Kontrastmittelaufnahme. Die DCE kann aber bei kleinen Karzinomen hilfreich sein, insbesondere wenn diese der DWI entgehen (z. B. unzureichendes SNR, zu niedrige b-Werte, Suszeptibilitätsartefakte). Areale mit früharterieller Kontrastmittelaufnahme können auch für das lokale Staging hilf- reich sein, weil sich Kapselüberschreitung und Samenblaseninvasion anhand der früharteriellen Kontrastmittelaufnahme darstellen.

\section{MR-Befundbilder}

Hauptaufgabe der mpMRT ist es, karzinomsuspekte Herdbefunde in der Prostata zu identifizieren und zu lokalisieren. Besonderes Augenmerk sollte dabei auf solchen Befunden liegen, die aufgrund ihrer Lokalisation und/oder Größe einer systematischen TRUS-Biopsie entgehen könnten, und daher eine zusätzliche gezielte Biopsie benötigen. Dies betrifft insbesondere ventral und apikal gelegene Karzinome.

\section{Merke}

Charakteristisch für karzinomsuspekte Herdbefunde sind:

- niedriges T2-Signal

- Diffusionseinschränkung mit hohem DWI-Signal bei hohem b-Wert und gleichzeitig niedrigem Signal auf der ADC-Parameterkarte

- früharterielle Kontrastmittelaufnahme [12].

Das Befundbild visualisiert die für Karzinome charakteristische Tumorbiologie („Cancer Hallmarks“), die durch eine erhöhte solide Zelldichte (Aufhebung der Drüsenarchitektur) sowie Neovaskularisation gekennzeichnet ist ( $>$ Abb. $\mathbf{3})$. 


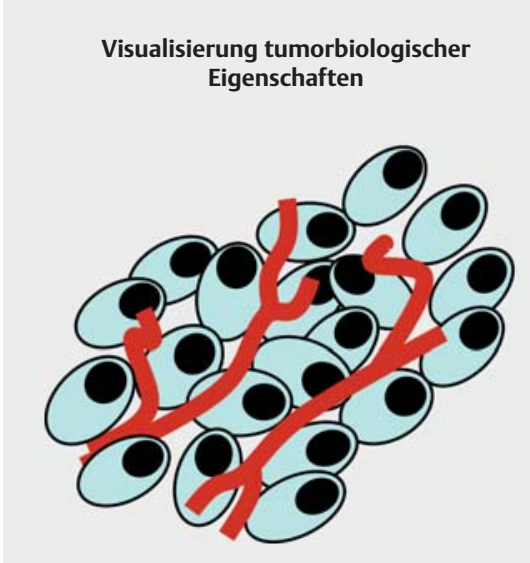

solides Wachstum, Neovaskularisation
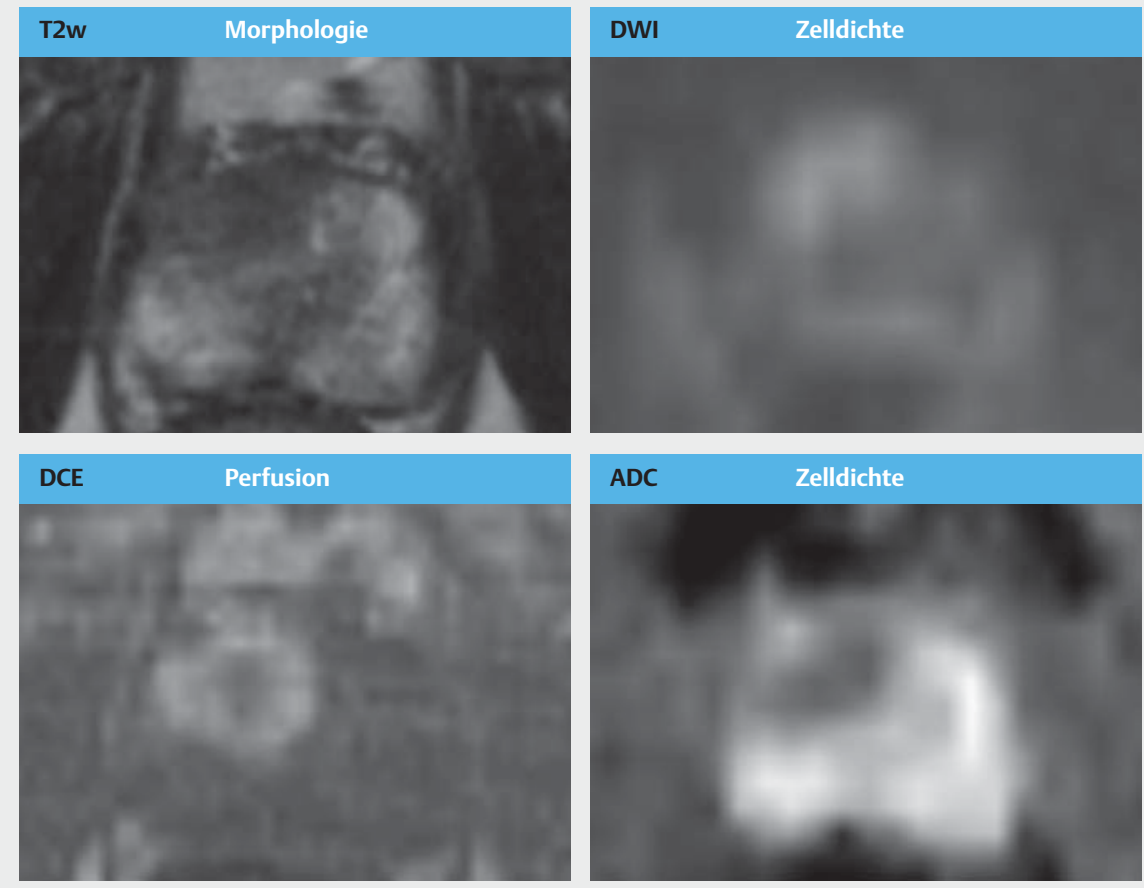

- Abb.3 Multiparametrische Prostata-MRT bei einem Prostatakarzinom. Das Karzinom ist biologisch durch typische „Cancer-Hallmarks“ gekennzeichnet (erhöhte Tumorzelldichte und verstärke Durchblutung aufgrund Neovaskularisation). Entsprechend ist die Kontrastdarstellung hypointens auf der T2w Aufnahme (Verlust des Drüsenaufbaus), hyperintens auf der DWI mit hohen b-Werten bzw. hypointens auf der ADCKarte (erhöhte Zelldichte). Bei der DCE ist eine frühe arterielle Kontrastmittelaufnahme nachzuweisen (Neovaskularisation). Die Befunde variieren aber bei den Patienten aufgrund der erheblich hohen biologischen Variabilität des Prostatakarzinoms.

Cave

Ein negativer MR-Befund kann niemals ausschließen, dass nicht doch kleine oder niedriggradige Karzinome vorhanden sind. Das betrifft insbesondere die Regionen der Prostata, die durch eine BPH oder Prostatitis signalverändert sind.

Für die Befundinterpretation ist es daher wichtig, auch die Abbildungseigenschaften der gutartigen Veränderungen zu kennen [13].

\section{Benigne Prostatahyperplasie}

Die BPH entwickelt sich in der TZ und kann zu unterschiedlichen, teilweise erheblichen Vergrößerungen der Drüse führen. Pathologisch kommt es zu einer knotigen Hyperplasie von Drüsen und Stroma in unterschiedlicher Zusammensetzung. Überwiegt der drüsige Anteil, ist die Signalgebung auf den T2w Aufnahmen mehr hyperintens, sonst mehr hypointens. Bei großen, rein stromalen Knoten kann die Unterscheidung von einem Karzinom sehr schwierig sein. Stromale BPHKnoten bestehen histologisch aus dichtem Stroma mit zudem vermehrten Kapillaren, was sich neben dem hypointensen Signal auf T2w Aufnahmen auch in Form einer reduzierten ADC bei eingeschränkter Diffusion und als auffällige DCE mit früharterieller Kontrastmit- telaufnahme zeigt. Das sind die gleichen Signaleigenschaften wie beim Prostatakarzinom.

\section{Merke}

Um stromale BPH-Knoten vom Karzinom abgrenzen zu können, nutzt man daher primär morphologische Charakteristika auf den T2w-Aufnahmen.

Die ZZ wird durch die BPH nach lateral verdrängt und kann an der Grenze von TZ und PZ als umschrieben $\mathrm{T} 2 \mathrm{w}$ hypointense Formationen abgegrenzt werden ( $\triangleright$ Abb. 2). Die Verwechslung mit einem Prostatakarzinom kann man vermeiden, indem man die TZ anhand ihrer typischen basisnahen und seitensymmetrischen Lokalisation identifiziert, wofür die koronaren T2w Aufnahmen hilfreich sind.

\section{Prostatitis}

Bei der Prostatitis finden sich in der PZ typischerweise flächige, glatt berandete und auf T2w Aufnahmen hypointense Bezirke, die zur Urethra hin spitz zulaufen (dreiecksförmige, flügelartige Form). Diese Veränderungen liegen häufig seitensymmetrisch vor. Die Kontrastmittelaufnahme ist in diesen Regionen verstärkt und ebenfalls flächig. Auffällige fokale Diffusionsstörungen liegen jedoch nicht vor. 
Die seltene granulomatöse Prostatitis kann bildmorphologisch nicht sicher von einem Prostatakarzinom abgegrenzt werden, da sie ebenfalls mit einer fokalen Diffusionseinschränkung und einer Mehrdurchblutung einhergeht.

Eine seltene Komplikation ist der Prostataabszess, der sich bildmorphologisch als typische, zentral einschmelzende Läsion mit entzündlichem Randsaum identifizieren lässt.

Die Entzündung der Samenblasen weist eine T2w hypointense Wandverdickung mit verstärkter Kontrastmittelaufnahme auf.

\section{Atrophie und Fibrose}

Atrophie und Fibrose der PZ sind ein diagnostisches Problem, weil sie sich als T2-hypointense Veränderungen darstellen. Zwar kann i.d. R. weder eine fokale Diffusionseinschränkung noch eine Mehrdurchblutung nachgewiesen werden, kleine und niedriggradige Karzinome sind aber trotzdem nicht sicher auszuschließen.

\section{Verkalkungen}

Verkalkungen der Prostata treten bei BPH, bei Entzündungen und als Folge eingedickten Sekretes auf. Makroskopische Verkalkungen stellen sich auf den MRAufnahmen als umschriebene hypointense Formationen dar. In den Drüsengängen eingedicktes und verkalktes Sekret verursacht die Bildung kleiner „Steine“ (Corpora amylacea). Diese Verkalkungen können sich auch feingranulär darstellen.

\section{Zysten}

Zysten sind anhand ihrer typischen Morphologie und Signalintensität leicht zu identifizieren. Sie sind unterschiedlich groß und haben - je nach ihrem Proteingehalt - unterschiedliche Signalintensitäten auf T1w und T2w Aufnahmen. Bei einer BPH kommen sie an verschiedenen Stellen vor. Eine solitäre Zyste liegt typischerweise im Bereich des Utriculus seminalis vor (Utrikuluszyste).

\section{Einblutungen}

Auf T1w Aufnahmen sind postbioptische Einblutungen flächige hyperintense Bezirke ohne raumfordernden Aspekt. Bei Hämatospermie stellt sich der Inhalt der Samenblasen - ebenfalls auf T1w Aufnahmen - flächig hyperintens dar.

\section{Prostatakarzinom}

Das Prostatakarzinom entsteht vorwiegend in der PZ und stellt sich hier als Herdbefund mit charakteristischem Signalverhalten dar:

\section{HINTERGRUNDWISSEN}

\section{Pathologische Einteilung}

Das Wachstumsmuster des Prostatakarzinoms wird anhand des Gleason Grade auf einer Skala von 1 (unauffällig, glanduläre Differenzierung) bis 5 (hochaggressiv, solide Entdifferenzierung) eingestuft. Für den Gleason-„Score“ werden das häufigste und das zweithäufigste oder das häufigste und niedrigste Differenzierungsmuster addiert. Von einem klinischen Prostatakarzinom spricht man erst ab einem pathologischen Score von $3+3$. Eine der derzeit größten Herausforderungen der pathologischen Diagnostik ist die Unterscheidung von klinisch signifikanten und klinisch nicht signifikanten Karzinomen [14]. Ein neues, auf dem Gleason-Score aufbauendes Gradingsystem versucht dem Rechnung zu tragen und unterscheidet nur noch 5 Gruppen, Grade-Group 1 - 5 [15].

- hypointenses T2w Signal

- hyperintenses DWI-Signal bei hohem b-Wert und hypointenses Signal auf der ADC-Karte

- schnelle früharterielle Kontrastmittelaufnahme

Entsprechend der hohen Variabilität der Tumoraggressivität findet sich in Abhängigkeit vom Gleason-Score und der Tumorheterogenität auch ein breites Spektrum der Abbildungseigenschaften.

Auch wenn eine Korrelation zwischen der ADC-Absenkung und dem Gleason-Score mehrfach nachgewiesen wurde, ist eine prospektive Zuordnung von ADC-Wert und Tumoraggressivität wegen zu hoher Überlappung der Messwerte kaum möglich. Die typischerweise ausgeprägte Tumorheterogenität lässt sich bei den unvermeidbaren Partialvolumeneffekten nicht ausreichend auflösen.

Wichtig zu betonen ist, dass ein negativer oder unspezifischer Befund der MRT ein Karzinom niemals ausschließt. Sehr kleine Karzinome entgehen völlig der Visualisierung. Kleine und niedriggradige Karzinome lassen sich nicht sicher von Prostatitis und Atrophie/ Fibrose abgrenzen. Kontrovers wird allerdings noch diskutiert, ob ein negativer MRT-Befund ein klinisch signifikantes Karzinom unwahrscheinlich macht. Doch die Evidenz steht hierfür noch aus.

Das Prostatakarzinom in der TZ ist viel seltener als in der PZ. In der TZ ist die Abgrenzung von Karzinomen gegenüber stromalen BPH-Knoten sehr schwierig bis unmöglich. Grund hierfür ist die oben beschriebene Histologie stromaler BPH-Knoten mit entsprechender Diffusionseinschränkung und Mehrdurchblutung. Die Einstufung bildmorphologisch suspekter Knoten stützt sich daher allein auf die Beurteilung der Knotenmorphologie auf den T2-betonten Aufnahmen: Ein runder, glatt berandeter Knoten ist demnach mit hoher Wahr- 


\section{FALLBEISPIEL (FORTSETZUNG)}

\section{Befund der mpMRT}

In der mpMRT bei 3,0 Tesla fanden sich in der PZ beidseits flächig T2-signalabgesenkte Bezirke mit früharteriell diffuser Kontrastmittelaufnahme ( $\mathbf{A} \mathbf{b} \mathbf{b} . \mathbf{4}$, obere Bilder). In diesen Bereichen waren in der Diffusionswichtung bei hohem b-Wert ( $\triangleright$ Abb. 4, links unten) und auf der ADC-Karte ( $\nabla$ Abb. 4 , rechts unten) beidseits multfokale, teils konfluierende Herdbefunde in der PZ zu erkennen. Die auf den T2w Aufnahmen und in der DCE flächig verteilten Signalveränderungen der PZ sind alleine als unspezifisch zu werten und könnten Ausdruck diffus entzündlicher Veränderungen sein. Auffällig war aber, dass in diesen Bereichen die DWI fokale Signalveränderungen mit unterschiedlich ausgeprägter Signalintensität zeigte.

Die transperineale MR/TRUS-Fusionsbiopsie (je 2 gezielte Probeentnahmen aus den auffälligen Regionen der PZ rechts und links sowie 1 systematische 24-fach-Sättigungsbiopsie) ergab ein Prostatakarzinom Gleason $3+4$ in allen 4 gezielt und in 4/24 der systematisch entnommenen Biopsieproben. Nach der roboterunterstützten radikalen Prostatektomie und der histologischen Aufarbeitung des Prostatektomiepräparates wurde die Diagnose „multifokales Prostatakarzinom beidseits, Stadium pT3a pN0 (0/8 Lymphknoten) R0, Gleason 3+4“ gestellt.
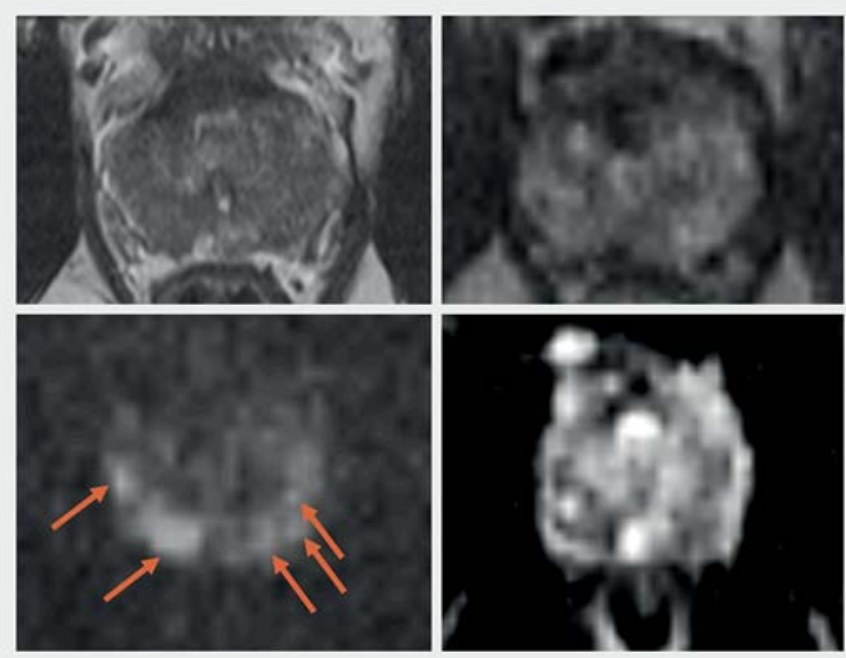

- Abb. 4 Multiparametrische MRT bei 3,0 Tesla in axialer Schichtführung in der T2w Turbo-Spin-Echo-Sequenz (oben links), T1w-GradientenEcho-Sequenz in der früharteriellen Phase (DCE) (oben rechts), der DWI $\left(b=1500 \mathrm{~s} / \mathrm{mm}^{2}\right)$ (unten links) und der ADC-Karte (unten rechts).

scheinlichkeit ein unauffälliger BPH-Knoten. Die Wahrscheinlichkeit eines Prostatakarzinoms steigt, wenn große, ovaläre, unscharf berandete Knoten mit zudem raumfordernden Aspekt vorliegen.

\section{PRAXISTIPP}

\section{Fallstricke}

Typische Pitfalls sind die ZZ, das anteriore fibromuskuläre Stroma und die granulomatöse Prostatitis:

- Die zentrale Zone ist ein umschriebenes, auf T2w Aufnahmen hypointenses Areal am Übergang von TZ (BPH) zu PZ und kann anhand der symmetrischen, basisnahen und dorsalen Lage identifiziert werden.

- Die AFS ist eine ebenso auf T2w Aufnahmen hypointenses Areal, das ventral und median symmetrisch liegt.

- Die granulomatöse Prostatitis ist ein typischer „Mimiker“ des Prostatakarzinoms. Die Erkrankung ist aber sehr selten und sollte nur bei typischer Anamnese (s. o.) in die Differenzialdiagnose einbezogen werden.

\section{Standardisierte Befundung PI-RADS-System}

Das „Prostate Imaging and Reporting System“ (PIRADS) wurde 2012 von der European Society of Urogenital Radiology (ESUR), basierend auf einer Initiative der AdMeTech Foundation's International Prostate MRI Working Group, in einer ersten Fassung (Version v1) publiziert. Schon bald darauf (2015) wurde es in Zusammenarbeit mit dem American College of Radiology (ACR) überarbeitet (Version v2), wobei viele praktisch wichtige Aspekte in Bezug auf eine Qualitätsverbesserung und Standardisierung berücksichtigt worden sind. Dazu gehörten u.a. die Minimalanforderungen an die Untersuchungsmethodik, ein System zur Kategorisierung der Wahrscheinlichkeit eines klinisch signifikanten Prostatakarzinoms sowie Empfehlungen für eine konsistente Befundkommunikation von Radiologen und Urologen. Enthalten ist auch ein hilfreiches Lexikon mit Begriffsdefinitionen, die im Befund standardisiert verwendet werden sollten. Handlungsempfehlungen für das weitere Prozedere wie bei BI-RADS für die Brustkrebsdiagnostik werden allerdings nicht gegeben. Im Folgenden wird nur auf PI-RADS v2 eingegangen, das Grundlage für die tägliche Routine sein sollte [16-18].

\section{Merke}

Das PI-RADS-System ist mittlerweile Bestandteil vieler wissenschaftlicher Publikationen und Grundlage für klinische Empfehlungen. 


\section{Erste Beurteilung von Prostata und Harnblase}

Am Anfang der Befundung sollte immer die Größe der Prostata bestimmt werden $($ Volumen = Produkt der 3 orthogonalen Durchmesser/2). Das Prostatavolumen wird benötigt, um die Höhe des PSA-Serumwertes in Relation hierzu zu beurteilen („PSA-Dichte“). Auch sollte man das Ausmaß der häufigsten gutartigen Veränderungen qualitativ beschreiben (BPH, Prostatitis, Atrophie/Fibrose). Da die BPH eine chronische Harnabflussstörung verursachen kann, sollte man auch die Harnblasenwand beurteilen, ob eine Balkenblase und/ oder Pseudodivertikel vorliegen, und einschätzen, ob die Urethermündungen aufgeweitet sind. Auch muss die innere Harnblasenwand nach Raumforderungen (Urothelkarzinom) durchmustert werden. In der flüssigkeitsgefüllten Harnblase sind polypöse Raumforderungen der Harnblasenwand auf den T2w Aufnahmen leicht zu erkennen.

\section{Beurteilung von Herdbefunden der Prostata}

Karzinomsuspekte Herdbefunde der Prostata werden in Bezug auf ein klinisch signifikantes Karzinom auf einer 5-Punkte-,,Likert-Skala“ eingestuft. Klinisch signifikant wird definiert als ein Befund mit pathologischem Gleason Score $>=7 a(3+4)$ oder $V>0,5$ cc oder T3a/b. Die Skala dient dazu, die Abbildungseigenschaften des Herdbefundes auf T2w Aufnahmen, DWI und DCE in der Zusammenschau zu bewerten und reicht von 1 = „sehr geringe Wahrscheinlichkeit für das Vorliegen eines klinisch signifikanten Karzinoms“ bis $5=$ „sehr hohe Wahrscheinlichkeit für das Vorliegen eines klinisch signifikanten Karzinoms“ ( $\triangleright$ Tab. 2).

\section{Merke}

Für die Kategorisierung dürfen nur die multiparametrischen Abbildungseigenschaften der Herdbefunde herangezogen werden, nicht aber andere Faktoren wie PSA-Serumwert oder DRU. PI-RADS ist eine Kategorisierung alleine des Bildbefundes.

Die endgültige klinische Entscheidung, ob - und wenn ja wie - eine Biopsie durchgeführt werden soll, hängt zudem von weiteren und davon unabhängigen Einflussgrößen ab.

Suspekte Befunde in der Prostata sind umschriebene Läsionen (Herdbefunde). Wie ihre Dignität aufgrund der multiparametrischen Kontraste beurteilt wird, hängt von ihrer anatomischen Zuordnung ab, d. h. davon, ob die Läsionen in der PZ oder in der TZ liegen: In der PZ liefert die DWI-Sequenz den führenden Kontrast, in der TZ die T2w Sequenz (jeweils sog. „dominante Sequenz“):
- Tab. 2 PI-Rads-Score. Die Wahrscheinlichkeit für das Vorliegen eines klinisch signifikanten Prostatakarzinoms wird auf einer 5-Punkte-„, LikertSkala“ angegeben.

\begin{tabular}{|c|l|}
\hline Stadium & Bedeutung \\
\hline PI-RADS 1 & $\begin{array}{l}\text { sehr geringe Wahrscheinlichkeit, d. h. ein Prostata- } \\
\text { karzinom ist sehr unwahrscheinlich }\end{array}$ \\
\hline PI-RADS 2 & $\begin{array}{l}\text { geringe Wahrscheinlichkeit, d. h. ein Prostatakarzinom } \\
\text { ist unwahrscheinlich }\end{array}$ \\
\hline PI-RADS 3 & $\begin{array}{l}\text { mittlere Wahrscheinlichkeit, d.h. der Befund ist unklar/ } \\
\text { mehrdeutig }\end{array}$ \\
\hline PI-RADS 4 & $\begin{array}{l}\text { hohe Wahrscheinlichkeit, d.h. ein Prostatakarzinom ist } \\
\text { wahrscheinlich }\end{array}$ \\
\hline PI-RADS 5 & $\begin{array}{l}\text { sehr hohe Wahrscheinlichkeit, d.h. ein Prostatakarzinom } \\
\text { ist sehr wahrscheinlich }\end{array}$ \\
\hline
\end{tabular}

- Karzinome der PZ kontrastieren sich aufgrund der eingeschränkten Diffusionskapazität (hohe Zelldichte) gegenüber dem normalen, nicht diffusionseingeschränkten Drüsengewebe.

- Karzinome der TZ sind aufgrund der Diffusionseigenschaft nicht von stromalen BPH-Knoten, d.h. benignen Befunden zu unterscheiden. Auch weisen diese Knoten eine typische früharterielle Kontrastmittelaufnahme auf. Suspekte Herdbefunde der TZ können daher nur auf Grundlage morphologischer Kriterien auf T2w Aufnahmen eingestuft werden, d.h. nach Form (irregulär), Berandung (unscharf, spikulär) und Binnenstruktur („Kohlestiftzeichen“ mit hervorstechend starker und homogener Signalabsenkung auf T2w Aufnahmen).

Die DCE spielt bei der Einstufung eine nur untergeordnete Rolle. Mehrdurchblutung ist eine unspezifische Gewebeeigenschaft, die nicht nur bei Karzinomen, sondern auch bei benignen Befunden wie Prostatitis und stromaler BPH vorliegt und bei niedriggradigen Karzinomen auch fehlen kann. Außerdem sind große und/oder aggressive Karzinome bereits anhand ihrer eindeutigen Diffusionseinschränkung zu identifizieren, und die Kenntnis der Mehrdurchblutung liefert keine klinisch relevante Zusatzinformation. Das Ergebnis der DCE wird nur qualitativ beurteilt und den beiden Kategorien „positiv“ (+,fokales früharterielles Enhancement vorhanden) und „negativ“ (-,fokales früharterielles Enhancement nicht vorhanden) zugeordnet. Aufwendige Datennachverarbeitungsroutinen und pharmakokinetische Modelle sind für die Auswertung der DCE nicht erforderlich. Ein früharterielles Enhancement kann auf den Einzelaufnahmen der dynamischen Serie erkannt werden, indem die Signalintensitäten von Läsion, umliegendem Gewebe und Femoralarterien im Zeitverlauf nach Kontrastmittelinjektion verglichen werden. Die DCE wird auch nur bei zweifelhaften (PIRADS 3) Befunden in der PZ herangezogen (s.u.). Für alle anderen Befunde sowohl in der PZ wie in der TZ 


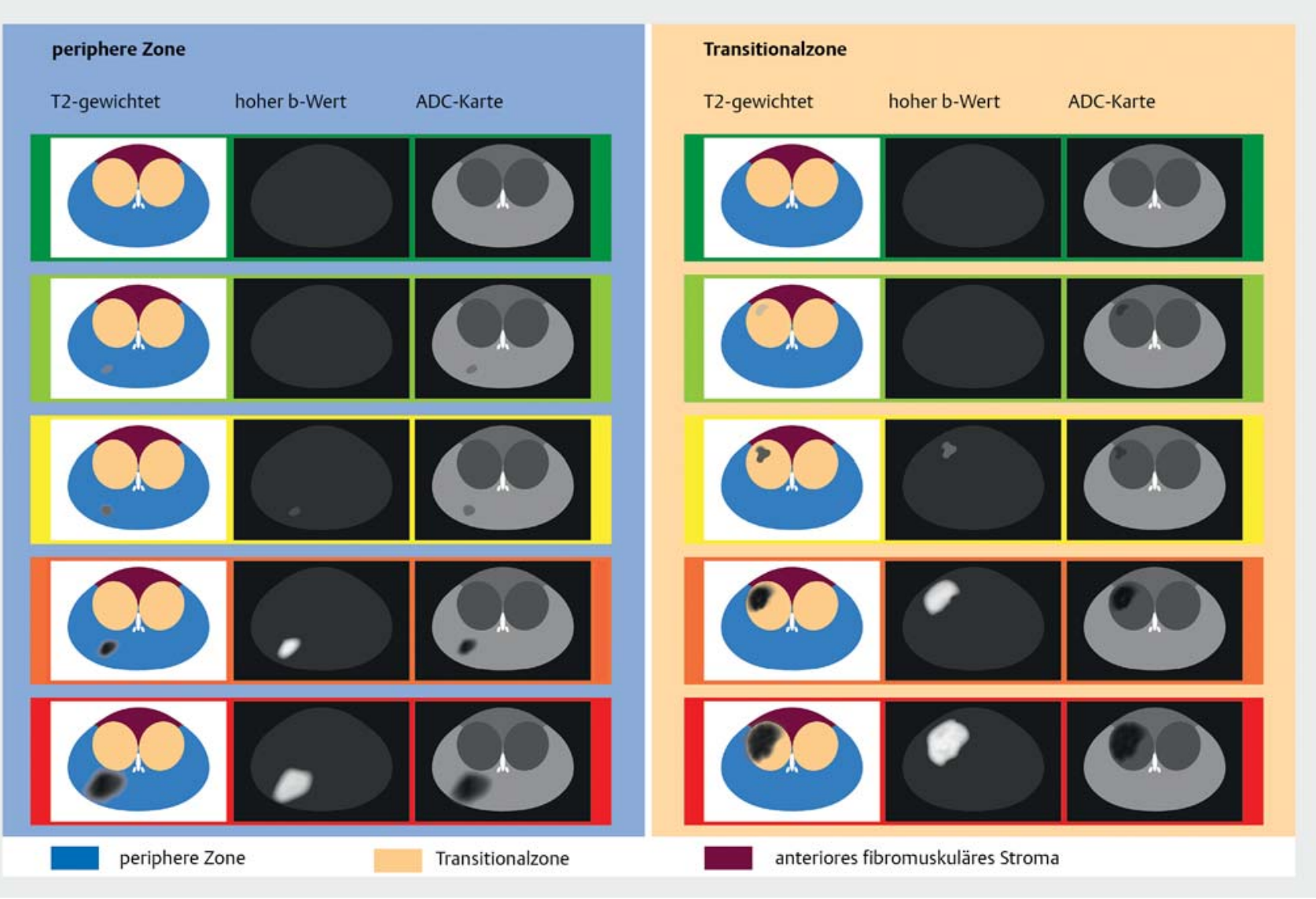

- Abb.5 PI-RADS-v2-Kategorisierung von mpMRT-Befunden auf einer 5-Punkte-Skala. In der peripheren Zone beruht die Kategorisierung auf der Signalintensität der DWI, in der Transitionalzone auf dem morphologischen Erscheinungsbild der T2w Aufnahme. Die DCE wird nur bei einem unklaren Befund in der peripheren Zone (PI-RADS 3) herangezogen und führt bei einer früharteriellen Kontrastmittelaufnahme zu einem Upgrading auf PI-RADS 4. Herdbefunde der Kategorie PI-RADS 4 und 5 unterscheiden sich nur dadurch, ob der Durchmesser größer oder kleiner $1,5 \mathrm{~cm}$ ist.

spielt das Ergebnis der DCE für die PI-RADS-Klassifikationen keine Rolle. Es muss jedoch betont werden, dass die DCE in Einzelfällen sehr wohl hilfreich ist, z. B. beim Nachweis kleiner aggressiver, d. h. klinisch signifikanter Karzinome. Diese Befunde können der DWI aufgrund ihrer Größe (zu geringes SNR) oder Lage (Suszeptibilitätsartefakte) entgehen. Das Erkennen eines früharteriellen Enhancements ist insbesondere von Bedeutung bei der Diagnostik der Kapselüberschreitung und/oder Samenblaseninvasion, bei der Rezidivdiagnostik sowie beim Active Surveillance.

Die Einordnung der multiparametrischen Befundkonstellationen in die jeweilige PI-RADS-Kategorien $\mathbf{1 - 5}$ sind für PZ und TZ getrennt in > Abb. 5 und $>$ Abb. 6 dargestellt:

- Die Kategorie PI-RADS 1 kennzeichnet einen vollständig unauffälligen Befund, was also bei älteren Männern alleine aufgrund der Häufigkeit gutartiger Erkrankungen praktisch nie vorkommt.

- Der Kategorie 2 zugeordnet werden BPH, (post)entzündliche Veränderungen, Atrophie und Fibrose, die sich mit unspezifischen Signalabsenkungen auf T2w Aufnahmen ohne jedoch auffällige Diffusionseinschränkung darstellen. Eine Ausnahme bilden stromale BPH-Knoten der TZ (s.o.).

- In die Kategorie PI-RADS 3 fallen nicht eindeutige Befunde bzw. Signalkonstellationen.

- Karzinomsuspekte Herdbefunde werden je nach Größe den Kategorien PI-RADS $4(\varnothing<1,5 \mathrm{~cm})$ oder PI-RADS $5(\varnothing>1,5 \mathrm{~cm})$ zugeordnet. In der PZ sind diese durch eine auffällige Diffusionseinschränkung gekennzeichnet, in der TZ durch eine auffällige Morphologie auf den T2w Aufnahmen.

Das PI-RADS-System kann mit einem Ampel-System verglichen werden: Benigne Befunde fallen in die Kategorien 1/2 (grün), unklare Befundkonstellationen in die Kategorie 3 (gelb) und maligne Befunde in die Kategorien $4 / 5$ (rot). 

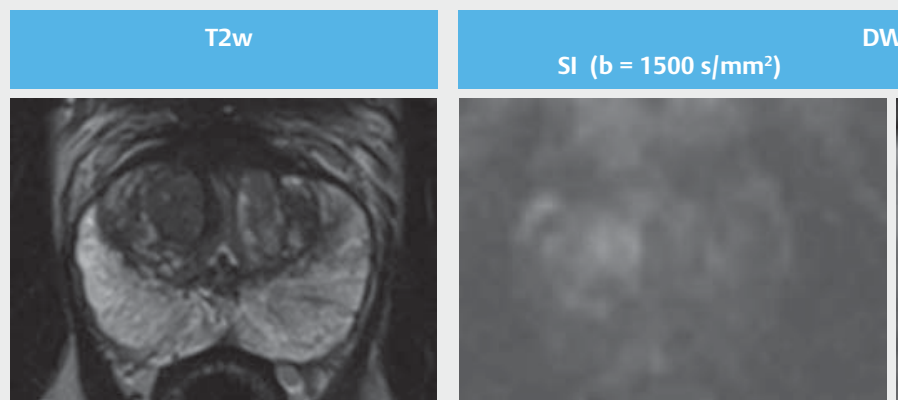

DWI

PI-RADS
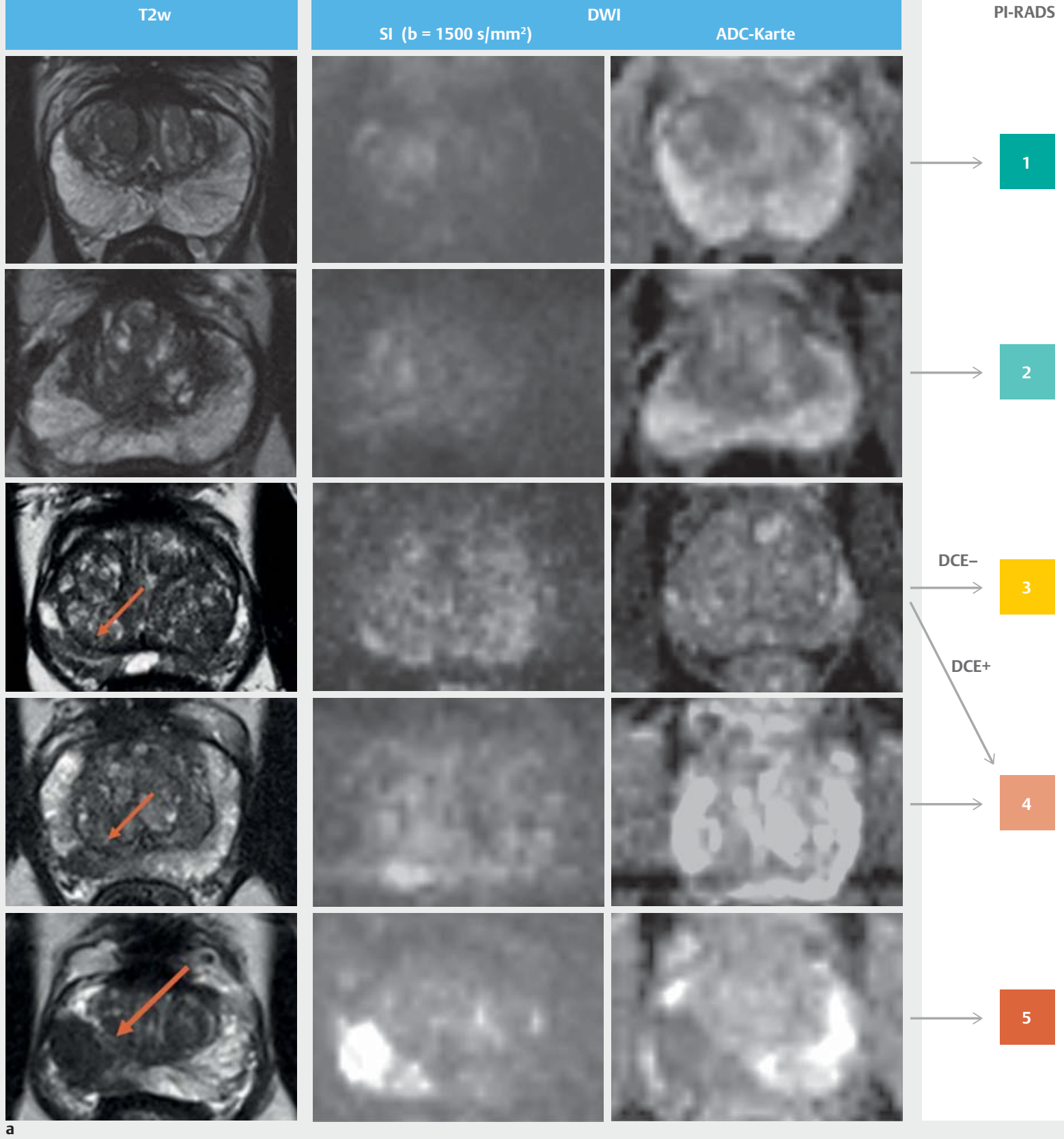

Abb. 6 Typische Befundmuster nach PI-RADS v2 in der peripheren Zone (PZ). Zu beachten ist, dass die häufigsten Karzinome in der peripheren Zone vorliegen und charakteristischerweise auf DWI/ADC zur Abbildung kommen ( $\geq$ PI-RADS 3). Die Bildqualität der DWI entscheidet damit die Qualität und klinische Relevanz des Befundes, da Urologen häufig ab PI-RADS 3 eine Biopsie in Betracht ziehen. a Typische Befundmuster nach PI-RADS v2 in der peripheren Zone (PZ). 1= Homogen hyperintense Signalgebung auf T2w Aufnahmen, kein Nachweis einer fokalen Signalabnormität auf DWI/ADC; 2 = Lineare, glatt berandete hypointense Formationen auf T2w Aufnahmen, leicht hypointense Darstellung auf ADC möglich; 3 = heterogene Signalabsenkung auf T2w Aufnahmen, leichte fokale Signalanhebung auf DWI und Signalabsenkung auf ADC; 4 = umschriebener Herdbefund von $<1,5 \mathrm{~cm}$ Durchmesser mit hypointensem Signal auf T2w Aufnahmen, fokale Signalanhebung auf DWI und Signalabsenkung auf ADC; 5 = wie 4 , aber mit Durchmesser $\geq 1,5 \mathrm{~cm}$. 

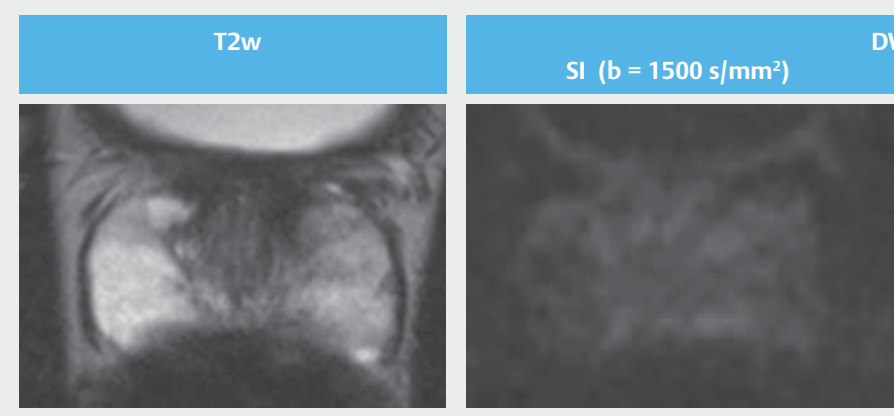

DWI
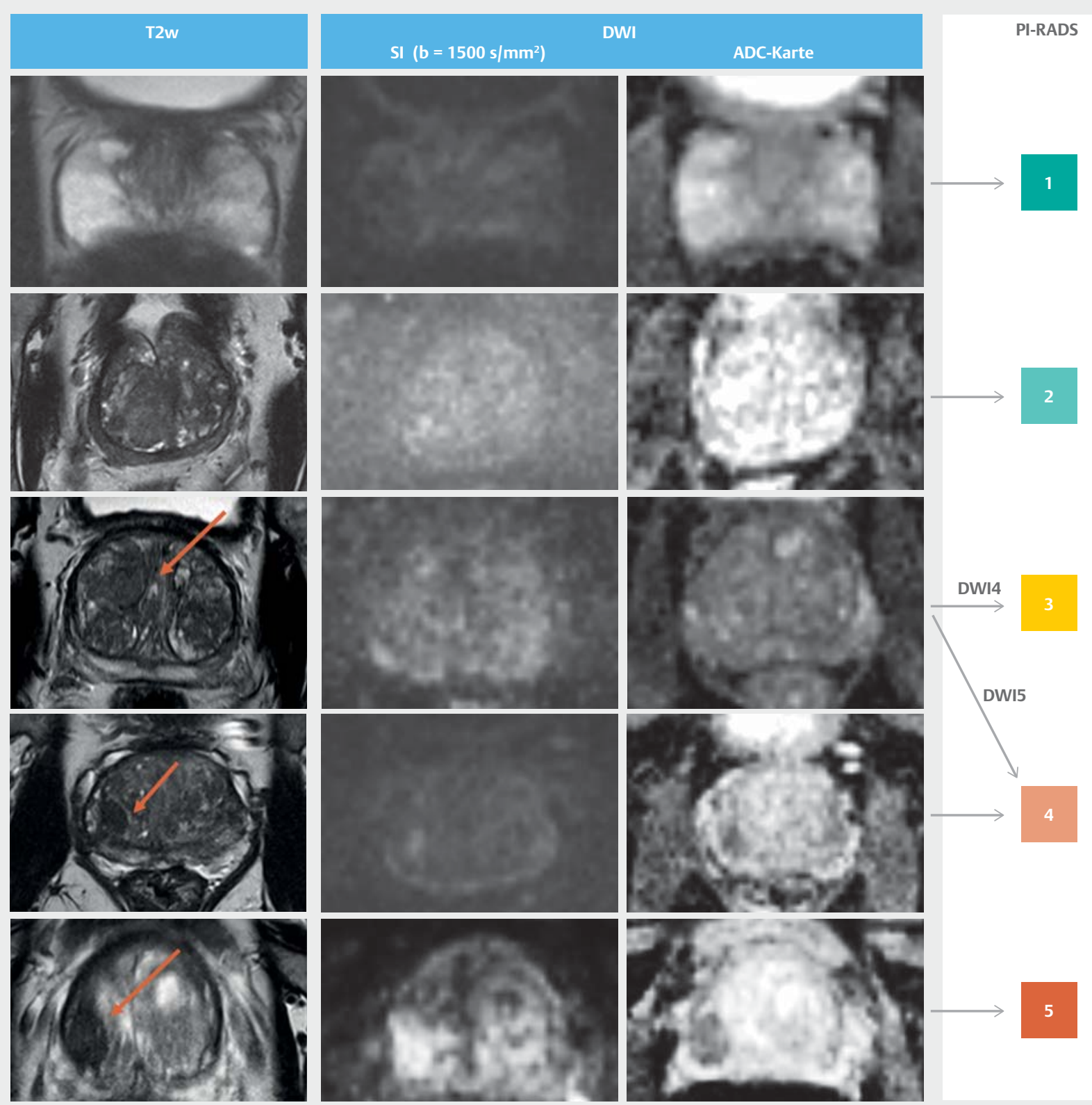

b

- Abb. 6 b Typische Befundmuster nach PI-RADS v2 in der Transitionalzone (TZ). 1 = homogene intermediäre Signalgebung auf T2w Aufnahmen, kein Nachweis einer fokalen Signalabnormität auf DWI/ADC; 2 = umschriebene, glatt berandete hypointense oder heterogene Formationen auf T2w Aufnahmen, leicht hypointense Darstellung auf ADC möglich; 3 = heterogene Signalabsenkung auf T2w Aufnahmen mit unscharfer Berandung, leichte fokale Signalanhebung auf DWI und Signalabsenkung auf ADC; 4 = ovalärer oder irregulärer Herdbefund von $<1,5 \mathrm{~cm}$ Durchmesser mit homogen oder heterogen hypointensem Signal auf T2w Aufnahmen, fokale Signalanhebung auf DWI und Signalabsenkung 


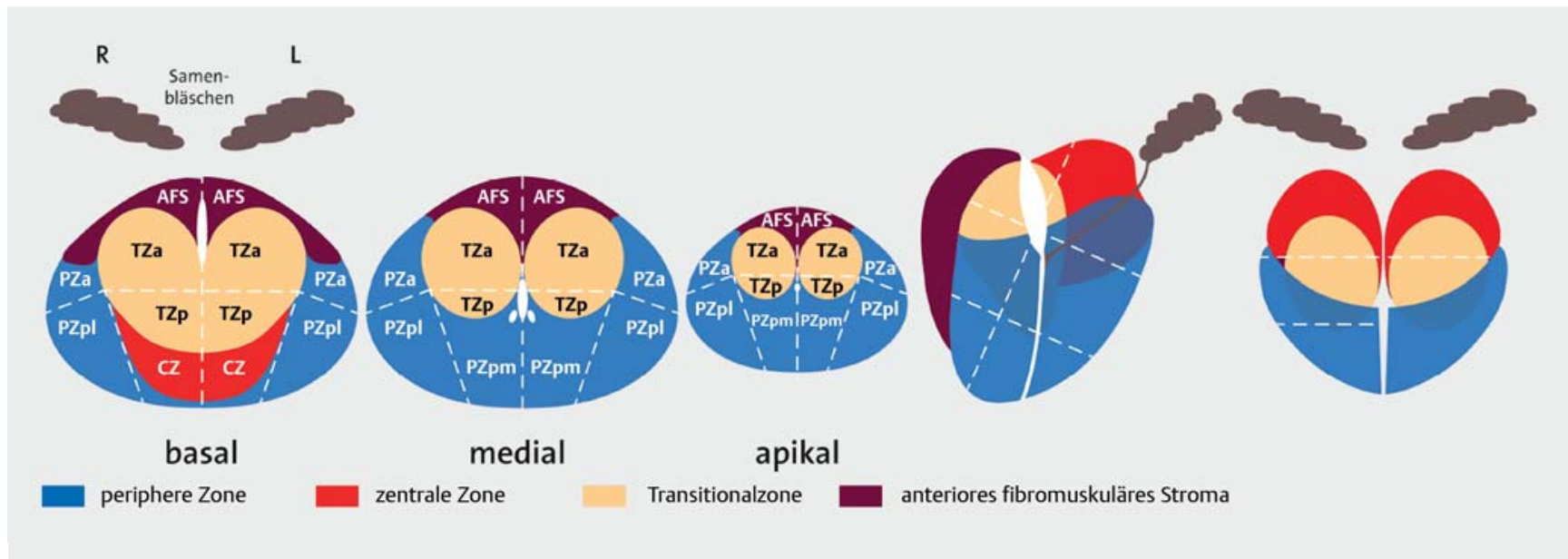

Abb.7 Befundschema der Prostata zur Markierung karzinomsuspekter Herdbefunde; AFS= anteriores fibromuskuläres Stroma, TZ=Transitionalzone, $\mathrm{PZ}$ = periphere Zone, $\mathrm{CZ}=$ zentrale Zone, $\mathrm{a}=$ anterior, $\mathrm{pl}=$ posterior lateral, $\mathrm{pm}=$ posterior medial.

Zur näheren Charakterisierung unklarer Herdbefunde (PI-RADS 3) gilt folgende Regel:

- Für die PZ wird die DCE herangezogen, wobei es ohne eine früharterielle Kontrastmittelaufnahme (d. h. DCE -) bei der Kategorie 3 bleibt, mit einer früharteriellen Kontrastmittelaufnahme (d. h. $\mathrm{DCE}+$ ) jedoch die Kategorie 4 gewählt wird.

- Für die TZ wird die DWI herangezogen, wobei nur bei einer großen (>1,5 cm) Diffusionseinschränkung auf die Kategorie PI-RADS 4 angehoben wird, und es sonst bei der Kategorie 3 bleibt.

\section{Sektorkarte}

Für den Urologen ist die exakte Lokalisation karzinomsuspekter Befunde in der Prostata von zentraler Bedeutung. Für die Befunddokumentation und -kommunikation wird in PI-RADS v2 ein Schema vorgeschlagen, das die Prostata in 39 Sektoren einteilt ( $\bullet$ Abb. 7). Markiert werden maximal 4 Läsionen mit den höchsten PI-RADSScores. Insgesamt sollen Läsionen mit einem PI-RADSScore von $\geq 3$ angegeben werden. Die sog. „Indexläsion" ist die mit dem höchsten PI-RADS-Score und dem größten Durchmesser oder einem organüberschreitenden Wachstum. Der schriftliche Befund sollte die Sektorkarte mit den markierten Befunden und der tabellarischen Auflistung der entsprechenden Sektorbezeichnungen und PI-RADS-Scores enthalten.

\section{Staging}

Bei histologisch gesichertem Karzinom kann die mpMRT auch zum T-Staging herangezogen werden ( Tab.3), wobei die Unterscheidung zwischen einem organbegrenzten (Stadien T1/2) und einem organüberschreitenden Tumorwachstum (Stadien T3a/T3b, T4) wesentlich ist. In diesem Zusammenhang ist auch die genaue anatomische Lage des Karzinoms wichtig, weil sie den möglichen Infiltrationsweg anzeigt-bei basaler Lage in die Samenblasen, bei mediolateraler Lage in das
- Tab. 3 TNM-Staging des Prostatakarzinoms.

\begin{tabular}{|l|l|}
\hline Stadium & Bedeutung \\
\hline Tumor & \\
\hline Tx & keine Aussage möglich \\
\hline T1a-c & $\begin{array}{l}\text { inzidentell gefundenes, kleines, nicht tastbares, bildgebend } \\
\text { nicht sichtbares Karzinom }\end{array}$ \\
\hline T2 & $\begin{array}{l}\text { auf die Prostata begrenztes Karzinom } \\
\text { - T2a:<50\% eines Seitenlappens }\end{array}$ \\
\hline & - T2b:>50\% eines Seitenlappens \\
\hline T3 & Karzinomausbreitung über die Prostatakapsel \\
\hline T4 & - T3a: über die Prostatakapsel \\
\hline Lymphknoten & Infiltration der Nachbarstrukturen \\
\hline Nx & keine Aussage möglich \\
\hline N0 & keine Metastasen in regionären Lymphknoten \\
\hline N1 & Metastasen in regionären Lymphknoten \\
\hline Metastasen & \\
\hline M0 & keine Fernmetastasen \\
\hline M1 & $\begin{array}{l}\text { Fernmetastasen nachweisbar } \\
\text { - M1a: in nicht regionären Lymphknoten }\end{array}$ \\
\hline & \\
\hline - M1c: in anderen Organen/Strukturen
\end{tabular}

neurovaskuläre Bündel und bei apikaler Lage in die extraprostatische Harnröhre. 

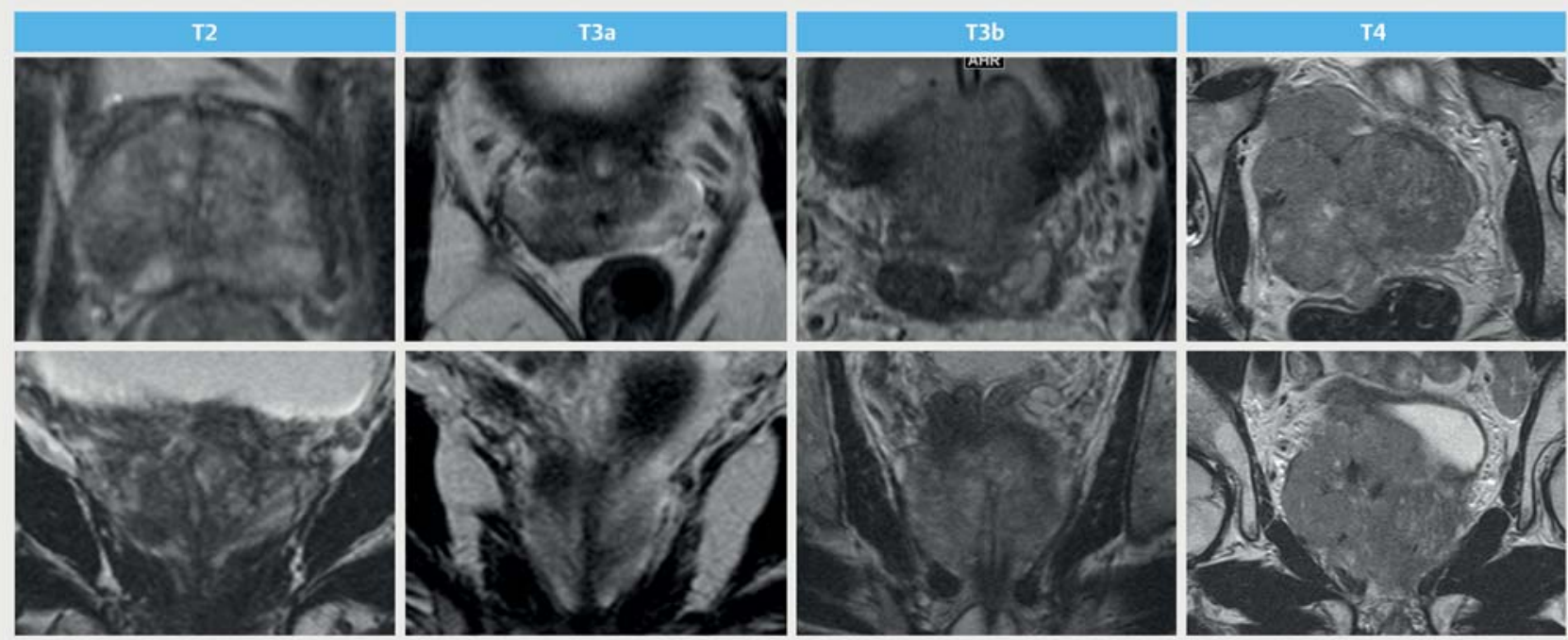

- Abb. 8 MR-basiertes T-Staging des Prostatakarzinoms. Von therapeutischer Relevanz ist vor allem die Trennung des organbegrenzten (T1/2) und des organüberschreitenden Tumorwachstums (T3/4). Bei einem organbegrenzten Wachstum ist zudem die genaue Angabe der Tumorlokalisation(en) sowie der Tumorausdehnung von Bedeutung für die Therapieplanung, insbesondere im Hinblick auf nerven- und kontinenzerhaltende Behandlungen. Liegt eine Organüberschreitung vor, wird bei zunehmend ungünstigerer Prognose zwischen Kapselüberschreitung (T3a), Samenblaseninfiltration (T3b) und Organinfiltration (T4) unterschieden. T4-Stadien werden seit der Einführung des PSATestes in den 1970er Jahren nur noch sehr selten beobachtet.

\section{Merke}

Das lokale T-Staging ist von außerordentlicher Relevanz für Prognoseabschätzung und Therapieplanung, sowohl hinsichtlich des onkologischen Erfolgs als auch der möglichen therapieassoziierten Morbidität (Inkontinenz, Impotenz).

Grundlage für das T-Staging sind die hochaufgelösten T2w Bilder, insbesondere zur Beurteilung der neurovaskulären Bündel und der Samenblasen. Eine Organüberschreitung ist durch entsprechende Befunde gekennzeichnet, wie z. B. Bulging der Prostataoberfläche, spikulierte Kontur der Prostataoberfläche, Obliteration der normalerweise auf T2w Aufnahmen aufgrund des Fettgewebes hyperintensen Randwinkel zwischen Prostata und Rektum bzw. zwischen Prostata und Samenblasen sowie hypointense Raumforderung in den Samenblasen ( $\triangleright$ Abb.8). Eine zusätzliche Diffusionsrestriktion erhärtet den Befund. Liegt der Verdacht auf ein organüberschreitendes Wachstum in das periprostatische Gewebe und/oder in die Samenblasen vor, kann die DCE hilfreich sein: Die Tumorinfiltration geht häufig mit charakteristischer früharterieller Kontrastmittelaufnahme einher.
Das Lymphknotenstaging ist mit der mpMRT nur sehr eingeschränkt möglich. Empfohlen wird, dass Lymphknoten mit einem Kurzachsendurchmesser von mehr als $8 \mathrm{~mm}$ als suspekt erwähnt werden sollten. Viele Studien haben aber belegt, dass im Frühstadium der Metastasierung häufig kleinere Lymphknoten befallen sind, und diese damit dem radiologischen Nachweis entgehen. Für die Beurteilung von Lymphknoten sollte daher neben der Größe unbedingt auch die Form, Binnenstruktur und das Enhancement herangezogen werden. Die DWI ist dabei nicht genau genug: Falsch negative Befunde finden sich bei kleinen Lymphknoten und falsch positive Befunde selbst bei größeren Lymphknoten aufgrund entzündlicher Veränderungen. Bei als suspekt eingestuften Lymphknoten ist die anatomische Lage von besonderer klinischer Relevanz. Die genaue Zuordnung zu den anatomischen Kompartimenten ist für das operative Vorgehen entscheidend, z. B. parailiakal (A. iliaca communis, externa, interna), obturatorisch, pararektal (innerhalb der mesorektalen Faszie), präsakral, femoral, paraaortal/parakaval. 


\section{FALLBEISPIEL (FORTSETZUNG) \\ Beurteilung}

Das Prostatakarzinom ist in etwa $60 \%$ der Fälle durch Multifokalität und biologische Heterogenität gekennzeichnet. Häufig ergibt sich auch ein Upgrading des Gleason-Scores nach Prostatektomie. Die DWI mit hohen b-Werten zeigte in dem Fall multiple und teils konfluierende Herdbefunde in der PZ beidseits mit unterschiedlicher Größe und Signalintensität, die auf ein multifokales Prostatakarzinom mit unterschiedlich großen Tumoranteilen und Zelldichten hindeuteten. Die MR-unterstützte Biopsie der suspekten Areale bestätigte in allen 4 Biopsiezylindern das Prostatakarzinom mit einem Gleason-Score von $3+4$. Die Sättigungsbiopsie lieferte keine zusätzliche klinisch relevante Information.
Bei diesem Patienten ermöglichte daher die Kombination aus mpMRT und MR/TRUS-Fusionsbiopsie die sichere Diagnose eines klinisch signifikanten multifokalen Prostatakarzinoms und stellte damit die individuelle Therapieindikation und Therapieplanung auf eine zuverlässige Grundlage. Neben der Multifokalität konnte auch mit nur wenigen, MR-basierten Gewebeentnahmen die prognostisch wichtige, individuelle Tumoraggressivität zuverlässig bestimmt werden. Der Gleason-Score der MR/TRUS-Fusionsbiopsie stimmte auch mit dem des Prostatektomiepräparates überein. Das pathologische Staging T3a zeigte eine Kapselinfiltration durch das Karzinom. Ein kapselüberschreitendes Karzinomwachstum zeigte sich jedoch MR-tomografisch nicht, und dementsprechend konnte auch eine R0-Resektion erzielt werden.

\section{KERNAUSSAGEN}

- Das Prostatakarzinom ist durch Multifokalität und biologische Variabilität gekennzeichnet. Die große Variationsbreite der Aggressivität erfordert eine differenzierte Risikoeinschätzung der Patienten.

- Die konventionelle urologische Diagnostik zur Früherkennung des Prostatakarzinoms umfasst die digitale rektale Untersuchung, den PSASerumtest und ggf. die systematische Prostatabiopsie. Diagnose, Grading und prognostische Einschätzung alleine auf Grundlage der konventionellen Diagnostik sind unbefriedigend.

- Der PSA-Test besitzt eine hohe Sensitivität bei allerdings geringer Spezifität. Erhöhte Serumwerte kommen auch bei den viel häufigeren gutartigen Prostataerkrankungen (BPH und Prostatitis) vor.

- Bei der Prostatabiopsie (meist als transrektale 12fach-TRUS-Biopsie) werden manche Karzinome nicht gesehen, weil sie z. B. klein sind, ventral oder apikal in der Prostata liegen oder weil aufgrund eines großen Prostatavolumens nur relativ wenig Gewebe untersucht wird.

- Pathologische Diagnose und individuelle Risikoeinschätzung beruhen auf dem pathologischen
„Gleason-Score“ im Material der Prostata-Stanzbiopsie oder der entfernten Prostata.

- Die (schwierige) Unterscheidung zwischen klinisch signifikanten und klinisch nicht signifikanten Karzinomen ist grundlegend für die individuell optimale Therapiewahl.

- Mit der mpMRT lässt sich die Diagnostik verbessern, die Prognose zuverlässiger einschätzen und die Therapie individualisieren. Sie kann mit modernen MR-Tomografen und ohne spezielles Equipment durchgeführt werden, erfordert aber:

- eine hohe Qualität und Standardisierung der Untersuchung

- eine ausreichend hohe Expertise bei der Bildbeurteilung

- eine standardisierte Befunddokumentation und -übermittlung

- die Zusammenarbeit von Urologen und Radiologen, um die Qualität nachhaltig zu kontrollieren und zu verbes-sern

- PI-RADS v2 ist ein von ESUR und ACR entwickelter Standard für die Objektivierung und Vereinheitlichung der Prostata-mpMRT. 
Interessenkonflikt

Der Autor gibt an, dass kein Interessenkonflikt besteht.

Über die Autoren

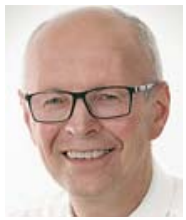

\section{Heinz-Peter Schlemmer}

Prof. Dr. med., Dipl.-Phys. 1980-1987 Studium der Physik in Heidelberg. 19871994 Studium der Medizin in Heidelberg. 1995-2000 Assistenzarzt in der Radiologie am DKFZ in Heidelberg. 2002 Habilitation. 2002-2010 Radiologische Universitätsklinik in Tübingen. Seit 2010 Leiter der Abteilung Radiologie des DKFZ in Heidelberg. Forschungsschwerpunkte: multiparametrische und multimodale onkologische Bildgebung, Bildgebung beim Prostatakarzinom u.a.

\section{Korrespondenzadresse}

Prof. Dr. med. Dipl.-Phys. Heinz-Peter Schlemmer Deutsches Krebsforschungszentrum

Abt. Radiologie

Im Neuenheimer Feld 280

69120 Heidelberg

E-Mail: h.schlemmer@dkfz.de

Danksagung

Mein besonderer Dank gilt Frau Dagmar Anders, DKFZ Heidelberg für ihre Mühe bei der Erstellung der Grafiken.

\section{Literatur}

[1] Fitzmaurice C, Allen C, Barber RM. Global Burden of Disease Cancer Collaboration. et al. Global, Regional, and National Cancer Incidence, Mortality, Years of Life Lost, Years Lived With Disability, and Disability-Adjusted Life-years for 32 Cancer Groups, 1990 to 2015: A Systematic Analysis for the Global Burden of Disease Study. JAMA Oncol 2016: DOI: 10.1001/jamaoncol.2016.5688

[2] Loeb S, Bjurlin MA, Nicholson J et al. Overdiagnosis and overtreatment of prostate cancer. Eur Urol 2014; 65: 1046 1055

[3] Rider JR, Sandin F, Andrén O et al. Long-term outcomes among noncuratively treated men according to prostate cancer risk category in a nationwide, population-based study. Eur Urol 2013; 63: 88-96

[4] Popiolek M, Rider JR, Andrén O et al. Natural history of early, localized prostate cancer: a final report from three decades of follow-up. Eur Urol 2013; 63: 428-435
[5] Bill-Axelson A, Garmo H, Holmberg L et al. Long-term distress after radical prostatectomy versus watchful waiting in prostate cancer: a longitudinal study from the Scandinavian Prostate Cancer Group-4 randomized clinical trial. Eur Urol 2013; 64: 920 - 928

[6] Giri VN, Beebe-Dimmer JL. Familial prostate cancer. Semin Oncol 2016; 43: $560-565$

[7] Pietro GD, Chornokur G, Kumar NB et al. Racial Differences in the Diagnosis and Treatment of Prostate Cancer. Int Neurourol J 2016; 20: (Suppl. 02): $112-119$

[8] Schröder FH. Review of diagnostic markers for prostate cancer. Recent Results Cancer Res 2009; 181: 173-182

[9] Aaron L, Franco OE, Hayward SW. Review of Prostate Anatomy and Embryology and the Etiology of Benign Prostatic Hyperplasia. Urol Clin North Am 2016; 43: 279-288

[10] Bhavsar A, Verma S. Anatomic imaging of the prostate. Biomed Res Int 2014; 2014: 728539

[11] Franiel T, Quentin M, Mueller-Lisse U et al. MRI of the Prostate: Recommendations on Patient Preparation and Scanning Protocol. Rofo 2017; 189: $21-28$

[12] Scheenen TW, Rosenkrantz AB, Haider MA et al. Multiparametric Magnetic Resonance Imaging in Prostate Cancer Management: Current Status and Future Perspectives. Invest Radiol 2015; 50: 594-600

[13] Kitzing YX, Prando A, Varol C et al. Benign Conditions That Mimic Prostate Carcinoma: MR Imaging Features with Histopathologic Correlation. Radiographics 2016; 36: 162 175

[14] Shah RB, Zhou M. Recent advances in prostate cancer pathology: Gleason grading and beyond. Pathol Int 2016; 66: $260-272$

[15] Epstein JI, Egevad L, Amin MB. Grading Committee. et al. The 2014 International Society of Urological Pathology (ISUP) Consensus Conference on Gleason Grading of Prostatic Carcinoma: Definition of Grading Patterns and Proposal for a New Grading System. Am J Surg Pathol 2016; 40: $244-252$

[16] European Society of Urogenital Radiology. ESUR Guidelines: Prostate MRI. Im Internet: http://www.esur.org/esurguidelines/prostate-mri/; Stand: 01.02.2017

[17] Hassanzadeh E, Glazer DI, Dunne RM et al. Prostate imaging reporting and data system version 2 (PI-RADS V2): a pictorial review. Abdom Radiol (NY) 2017; 42: 278 -289

[18] Vargas HA, Hötker AM, Goldman DA et al. Updated prostate imaging reporting and data system (PIRADS v2) recommendations for the detection of clinically significant prostate cancer using multiparametric MRI: critical evaluation using whole-mount pathology as standard of reference. Eur Radiol 2016; 26: 1606 - 1612

Bibliografie

DOI http://dx.doi.org/10.1055/s-0042-122604

Radiologie 2017; 17: 43-60

(c) Georg Thieme Verlag KG Stuttgart · New York ISSN 1616-0681 


\section{Punkte sammeln auf CME.thieme.de}

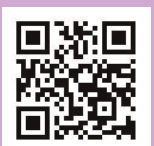

Diese Fortbildungseinheit ist 12 Monate online für die Teilnahme verfügbar.

Sollten Sie Fragen zur Online-Teilnahme haben, finden Sie unter http://cme.thieme.de/hilfe eine ausführliche Anleitung. Wir wünschen viel Erfolg beim Beantworten

der Fragen!

Unter eref.thieme.de/ZZWHP84 oder über den QR-Code kommen Sie direkt zum Artikel zur Eingabe der Antworten.

VNR 2760512017152372746

\section{Frage 1}

Welche der folgenden Aussagen zur Epidemiologie des Prostatakarzinoms ist richtig?

A Das Maximum der altersabhängigen Erkrankungshäufigkeit liegt bei 55 Jahren.

B Es ist in den Industrienationen die Krebserkrankung mit der größten Mortalität.

C In Deutschland stehen Inzidenz und Mortalität einem Verhältnis von etwa $3: 1$.

D Eine familiäre Häufung ist nicht nachgewiesen.

E Das Prostatakarzinom zeichnet sich besonders durch Multifokalität und biologische Variabilität aus.

\section{Frage 2}

Welche der folgenden Aussagen zur konventionellen Diagnostik des Prostatakarzinoms ist nicht richtig?

A Ein suspekt erhöhter PSA-Serumwert kann auch bei BPH und Prostatitis vorliegen.

B Zur Diagnostik des Prostatakarzinoms wird üblicherweise eine systematische 6-fach-TRUS-Stanzbiopsie durchgeführt.

C Der Schwellenwert, ab dem eine PSA-Serumkonzentration als karzinomsuspekt eingestuft wird, ist altersabhängig festgelegt.

D Die konventionelle urologische Diagnostik zur Früherkennung des Prostatakarzinoms setzt sich aus digitaler rektaler Untersuchung, PSA-Serumtest und ggf. systematischer Prostatabiopsie zusammen.

E Auch der 12-fach-TRUS-Randombiopsie können ventral gelegene Prostatakarzinome entgehen.

\section{Frage 3}

Welche der folgenden Aussagen zur Anatomie der Prostata ist richtig?

A Die beiden Ductus deferentes sind in der Prostata von der Transitionalzone umgeben.

B Die zentrale Zone ist der Entstehungsort der BPH.

C Die periphere Zone befindet sich vorwiegend dorsal/lateral und nimmt zum Apex hin an Volumen zu.

D Die periphere Zone enthält ca. $60 \%$ und die zentrale Zone ca. $40 \%$ der Drüsenanteile.

E Die Zone fibromuskulären Stromas liegt vor allem ventral apikal.

\section{Frage 4}

Welche Erkrankung der Prostata ist am seltensten?
A Adenokarzinom
B Duktales Karzinom
C BPH
D Prostatitis
E Atrophie

\section{Frage 5}

Welche der folgenden Aussagen zur DWI ist richtig?

A Die DWI-Sequenz muss mit mindestens 5 unterschiedlichen b-Werten durchgeführt werden.

B Kleine Karzinome können mit DWI zuverlässiger nachgewiesen werden als mit DCE.

C DCE und DWI liefern gleichwertige Informationen.

D Der ADC-Wert korreliert mit dem Gleason-Score, wenn auch große Überlappungsbereiche beobachtet werden.

E Der ADC-Wert ist von besonderer Bedeutung für den Nachweis von Karzinomen der TZ. 


\section{Punkte sammeln auf CME.thieme.de}

Fortsetzung ...

\section{Frage 6}

Welche der folgenden Aussagen zur mpMRT der Prostata ist richtig?

A Im Fall einer vorangegangenen Prostatabiopsie sollte ein Intervall von mindestens 3 Monaten eingehalten werden.

B Eine i.v. Gabe von Antiperistaltika ist obligat.

C Luft im Rektum kann die Qualität der Untersuchung negativ beeinflussen.

D Die Untersuchung muss zwingend bei 3,0 Tesla durchgeführt werden.

E Die Verwendung einer Endorektalspule ist i.d. R. erforderlich.

\section{Frage 7}

Welche der folgenden Informationen ist zur Beurteilung einer Prostata-MRT am wenigsten erforderlich?

A PSA-Serumwert und PSA-Dynamik

B Anzahl, Zeitpunkt und Ergebnis vorangegangener Prostatabiopsien

C Schmerzanamnese

D Tastbefund der rektalen Untersuchung

E Familienanamnese

\section{Frage 8}

Welche der folgenden Aussagen zur DCE ist nicht richtig?

A Die Untersuchung kann in der Routine durchgeführt werden.

B Die DCE ist hilfreich für das T-Staging.

C Prostatakarzinome zeichnen sich immer durch eine früharterielle Kontrastmittelaufnahme aus.

D Die Berechnung quantitativer pharmakokinetischer Parameter ist entscheidend für die Bildinterpretation.

E Die DCE kann mit handelsüblichem Gd-haltigem Kontrastmittel durchgeführt werden.

\section{Frage 9}

Welche der folgenden Aussagen zur PI-RADS-Klassifikation ist nicht richtig?

A PI-RADS 3 kennzeichnet unklare Befunde.

B PI-RADS 4 und 5 unterscheiden sich alleine durch die Größe des Läsionsdurchmessers.

C PI-RADS gibt keine Handlungsanweisungen für das weitere diagnostische und therapeutische Vorgehen.

D Bei PI-RADS 1 ist ein Prostatakarzinom ausgeschlossen.

E Eine Prostatabiopsie wird häufig ab PI-RADS $\geq 3$ erwogen.

\section{Frage 10}

Welche der folgenden Aussagen zu den Kontrasten einer mpMRT ist nicht richtig?

A Das Ergebnis der DCE spielt eine nur untergeordnete Rolle bei PI-RADS v2.

B Karzinomsuspekte Befunde der peripheren Zone haben eine hyperintenses Signal auf den diffusionsgewichteten Bildern mit hohen b-Werten.

C Stromale BPH-Knoten stellen sich mit Diffusionseinschränkung dar.

D Ein Karzinom der Transitionalzone lässt sich aufgrund der charakteristischen früharteriellen Kontrastmittelaufnahme mit Wash-out sicher diagnostizieren.

E Karzinomsuspekte Befunde der Transitionalzone werden bei PI-RADS v2 alleine aufgrund von morphologischen Parametern auf T2w Aufnahmen eingestuft. 